The deflection is zero at $x=0$ and $x=l$. The application of these conditions to (8) yields

$$
C_{1}=-\frac{P}{12 b}\left(6 b^{3}-3 a^{2} b+a^{3}\right), \quad C_{2}=0 .
$$

If, in addition to concentrated loads, the beam carries loads which are uniformly distributed over certain sections, the treatment is similar.

\title{
A VARIATIONAL PRINCIPLE FOR A STATE OF COMBINED PLASTIC STRESS*
}

\section{By G. H. HANDELMAN $\dagger$ (Brown University)}

In a recent paper ${ }^{1} M$. A. Sadowsky has stated a heuristic principle of maximum plastic resistance which he has applied to several states of combined plastic stress. The principle states that "among all statically possible stress distributions (satisfying all three equations of equilibrium, the condition of plasticity, and boundary conditions), the actual stress distribution in plastic flow requires a maximum value of the external effort necessary to maintain the flow." W. Prager, in a contribution to the discussion of this paper ${ }^{2}$, has shown that the principle can be so interpreted as to lead to the correct differential equation for a beam under combined torsion and tension. This note is concerned with an accurate statement of the principle together with a proof of its validity for the case of a beam in a perfectly plastic state under combined torsion and bending by couples, the cross-section of the beam having an axis of symmetry. Specifically, we shall prove the following variational principle for such a system.

Among all statically possible stress distributions in a beam under a given torque (satisfying the equations of equilibrium, the condition of plasticity, and boundary conditions), the actual stress distribution when plastic flow occurs is the one for which the bending moment is stationary.

Let us choose the coordinate axes in the following fashion. $y$ lies along the axis of symmetry of the cross-section, $z$ passes through the center of gravity of the crosssection and is parallel to the generators of the cylindrical beam, and $x$ is perpendicular to $y$ and $z$. We assume that the strain velocities, $v_{x}, v_{y}, v_{z}$, are given by the same expressions as in the case of an incompressible elastic material; i.e.,

$$
\begin{aligned}
& v_{x}=-\omega y z+\frac{1}{2} \theta x y, \\
& v_{y}=\omega x z-\frac{1}{4} \theta\left(x^{2}-y^{2}-2 z^{2}\right), \\
& v_{z}=\omega w(x, y)-\theta y z .
\end{aligned}
$$

* Received Sept. 9, 1943.

$\dagger$ This note was prepared at the suggestion of Professor W. Prager while the author was a participant in the Program of Advanced Instruction and Research in Mechanics at Brown University and was presented to the American Mathematical Society on Sept. 12, 1943 under the title of On a principle of M. A. Sadowsky.

1 M. A. Sadowsky, Journal of Applied Mechanics 10, A-65 (1943).

2 Journal of Applied Mechanics 10, A-238 (1943). 
$\omega$ and $\theta$ are constants ( $\omega=$ angle of twist per unit length per unit time) and $w(x, y)$ is an unknown function. The components of the strain velocity tensor, which are

etc., will then be

$$
\epsilon_{x y}=\frac{1}{2}\left(\frac{\partial v_{x}}{\partial y}+\frac{\partial v_{y}}{\partial x}\right),
$$

$$
\begin{array}{lll}
\epsilon_{x x}=\frac{\theta}{2} y, & \epsilon_{y y}=\frac{\theta}{2} y, & \epsilon_{z z}=-\theta y, \\
\epsilon_{x y}=0, & \epsilon_{x z}=\frac{\omega}{2}\left(\frac{\partial w}{\partial x}-y\right), & \epsilon_{y z}=\frac{\omega}{2}\left(\frac{\partial w}{\partial y}+x\right) .
\end{array}
$$

The components of the stress tensor will be denoted by $\sigma_{x x}, \sigma_{x y}$, etc. The material is assumed to be incompressible and perfectly plastic. The stress-strain relations can therefore be written in the form

$$
\begin{aligned}
\left(\sigma_{x x}-\sigma_{y y}\right):\left(\sigma_{y y}-\sigma_{z z}\right):\left(\sigma_{z z}-\sigma_{x x}\right): \sigma_{x y}: \sigma_{y z}: \sigma_{z x} \\
=\left(\epsilon_{x x}-\epsilon_{y y}\right):\left(\epsilon_{y y}-\epsilon_{z z}\right):\left(\epsilon_{z z}-\epsilon_{x x}\right): \epsilon_{x y}: \epsilon_{y z}: \epsilon_{z x} .
\end{aligned}
$$

In addition the following yield condition must be fulfilled:

$$
\left(\sigma_{x x}-\sigma_{y y}\right)^{2}+\left(\sigma_{y y}-\sigma_{z z}\right)^{2}+\left(\sigma_{z z}-\sigma_{x x}\right)^{2}+6\left(\sigma_{x y}^{2}+\sigma_{y z}^{2}+\sigma_{x z}^{2}\right)=6 k^{2},
$$

where $k$ is a given constant. Eq. (1) will be satisfied if the stress tensor has the form

$$
\begin{aligned}
\sigma_{x x}=\sigma_{y y}=\sigma_{x y}=0, & \sigma_{x z}=\mu \omega\left(\frac{\partial w}{\partial x}-y\right), \\
\sigma_{y z}=\mu \omega\left(\frac{\partial w}{\partial y}+x\right), & \sigma_{z z}=-3 \theta \mu y,
\end{aligned}
$$

$\mu=\mu(x, y)$ being an unknown function of $x$ and $y$. The equations of equilibrium for such a stress system reduce to

$$
\frac{\partial \sigma_{x z}}{\partial x}+\frac{\partial \sigma_{y z}}{\partial y}=0 .
$$

Consequently we may introduce a stress function $k u(x, y)$ such that

$$
\sigma_{x z}=k \frac{\partial u}{\partial y}, \quad \sigma_{y z}=-k \frac{\partial u}{\partial x} .
$$

Since the surface of the beam is not stressed, $u=$ const. on the surface. For convenience, this constant may be taken as zero. Combining Eq. (4) and the yield condition, Eq. (2), we have

$$
\sigma_{z z}=k \sqrt{ } 3\left(1-u_{x}^{2}-u_{y}^{2}\right)^{1 / 2},
$$

where $u_{x}=\partial u / \partial x, u_{y}=\partial u / \partial y$. Therefore the function $\mu$ must be given by

$$
\mu=\frac{-k}{y \theta \sqrt{3}}\left(1-u_{x}^{2}-u_{y}^{2}\right)^{1 / 2} .
$$


By means of Eq. (3) and Eq. (4), we can compute $\partial w / \partial x$ and $\partial w / \partial y$ in terms of the stress function $u$. This will yield

$$
\frac{\partial w}{\partial x}=\frac{k}{\omega \mu} u_{y}+y, \quad \frac{\partial w}{\partial y}=\frac{-k}{\omega \mu} u_{x}-x .
$$

Taking cross derivatives and subtracting one finds

$$
\frac{\partial}{\partial y}\left(\frac{1}{\mu} u_{y}\right)+\frac{\partial}{\partial x}\left(\frac{1}{\mu} u_{x}\right)+\frac{2 \omega}{k}=0
$$

which becomes by virtue of Eq. (5)

$$
\frac{\partial}{\partial x}\left[y u_{x}\left(1-u_{x}^{2}-u_{y}^{2}\right)^{-1 / 2}\right]+\frac{\partial}{\partial y}\left[y u_{y}\left(1-u_{x}^{2}-u_{y}^{2}\right)^{-1 / 2}\right]-\frac{2 \omega}{\theta \sqrt{3}}=0 .
$$

We shall now determine the Euler equation for the variational principle stated previously. The bending moment and torque are given by

$$
\begin{aligned}
\text { Bending Moment } & =\iint \sigma_{z z} y d x d y=k \sqrt{3} \iint y\left(1-u_{x}^{2}-u_{y}^{2}\right)^{1 / 2} d x d y, \\
\text { Torque } & =2 k \iint u d x d y .
\end{aligned}
$$

The domain of integration is the cross section of the beam. We note that the symmetry assumption has been used in writing the bending moment in the form above. According to the usual procedure, let us form the function

$$
\varphi\left(u, u_{x}, u_{y}\right)=y\left(1-u_{x}^{2}-u_{y}^{2}\right)^{1 / 2}+\lambda \mu,
$$

where $\lambda$ is an unknown constant. The Euler equation can then be written as

$$
\frac{\partial}{\partial x}\left(\frac{\partial \varphi}{\partial u_{x}}\right)+\frac{\partial}{\partial y}\left(\frac{\partial \varphi}{\partial u_{y}}\right)-\frac{\partial \varphi}{\partial u}=0 .
$$

Substituting for $\varphi$ from Eq. (7), one finds

$$
\frac{\partial}{\partial x}\left[y u_{x}\left(1-u_{x}^{2}-u_{y}^{2}\right)^{-1 / 2}\right]+\frac{\partial}{\partial y}\left[y u_{y}\left(1-u_{x}^{2}-u_{y}^{2}\right)^{-1 / 2}\right]+\lambda=0 .
$$

The unknown constant $\lambda$ is determined by the fact that the torque is prescribed. On the other hand, $\omega$ and $\theta$ can not be given arbitrarily but must be found from exactly the same condition. Consequently Eq. (8) is the same as Eq. (6), which proves the variational principle. 